\title{
The German Bunsen Society in Vienna.
}

\author{
By Prof. Fritz Paneth (Königsberg i. Pr.).*
}

$\mathrm{T}$ HE German Bunsen Society for Applied Physical Chemistry and Electrochemistry holds a general meeting annually, and this year the meeting took place in Vienna on May 25-28. Some 350 physicists and chemists from Germany and Austria attended the meeting and, as is usually the case, there were present a number of representatives from several other countries, including Switzerland, Czecho-Slovakia, Hungary, Poland, Holland, Sweden, Spain, the United States of America, and Great Britain. From the lastnamed country no less than eight well-known chemists were present.

In the Bunsen Society it is customary to fix upon a main topic for the lectures, and this principle was again adopted. Last year the main topic was of an essentially theoretical nature, so that this year, in view of the fact that the Bunsen Society is intended to serve the interests also of applied physical chemistry, a topic of importance in technical work was chosen as the subject for discussion. The title was "Advancess in the Science of Metals, and their Applications to Light Metals ". The president of the Bunsen Society had invited five members to give lectures in the nature of a comprehensive survey on the selected theme, and these included Prof. G. Tammann, who celebrated his seventieth birthday during the meeting on May 28, and was the object of numerous expressions of esteem. Amongst others, there was an address written by Prof. Nernst, which was presented to him during the first session by the president of the Society, and in which his great services to the most divers branches of physical chemistry were emphasised.

The first comprehensive survey was given by Prof. R. Becker (Berlin) on "Electrical and Magnetic Properties of Metals". He commenced by pointing out that the old classical theory, which treated as a gas the whole of the free electrons present in a metal, is entirely inadequate in the face of two facts of experience ; it is unable to explain either why the electrical resistance vanishès with diminishing temperature, or why the free electrons make no contribution to the specific heat. Both points are cleared up by two profound alterations which have to be made to the classical picture, according to quantum mechanics. The individual electron is not to be described as a point charge, but as a wave function, and the interference of the electron thus rendered possible brings about the disappearance of the resistance in a lattice which is not disturbed by thermal movement. On the other hand, Pauli's principle (Fermi statistics) becomes effective in the sense that the free electrons are unable to supply any appreciable contribution to the specific heat. The practical application of the quantum mechanical picture of the metallic state envisaged by modern

* Translated by Dr. R. W. Lawson. theoretical physics is, for the time being, however, held up by mathematical difficulties.

The next lecture, by Dr. G. Masing (Berlin), was entitled "Age-Hardening, especially on the Basis of Experience with Light Metals and with Alloys of Beryllium ". Masing started off from the effect discovered by Wilm in 1909 with duralumin, in which a marked hardening (Vergütung) of alloys can be attained by quenching the material from a high temperature, and afterwards storing it at ordinary temperatures. This phenomenon, which has been observed not only with alloys of aluminium, but also with alloys of copper and other heavy metals with beryllium or silicon, as well as with several varieties of steel, etc., has not hitherto found a wholly satisfactory explanation. It is certain that a necessary condition for the hardening is that supersaturated mixed crystals are produced by the quenching; hence we must assume that the hardening is somehow associated with an alteration of these supersaturated mixed crystals. On the basis of all the available evidence, especially the results of X-ray investigations, Dr. Masing was of the opinion that the hardening can be regarded generally as an accompaniment of the separating out of a second type of erystal from a mixed crystal. The details of this segregative process, and accordingly also of age-hardening, are probably different, however, for different alloys.

The third speaker was Prof. Tammann (Göttingen), on the subject of "Recrystallisation". It is well known that the structure of a specimen of metal can be fundamentally altered by rolling or forging. This is due to the fact that the crystallites become drawn out into plates or fibres by slipping on defined crystallographic planes. In this way the originally random orientation of the crystallites passes over into a more or less completely ordered form. The physical and chemical properties of the metal, as well as its elastic properties, change in this process, and we may thus assume that, as a result of the displacement along the slip-planes, an alteration takes place also in the atoms themselves. If we increase the temperature of the specimen, the original properties tend to return, and in every metal this recovery of the crystallites takes place within a different range of temperature. With further increase of temperature, fresh boundaries and individual small grains, which can be detected by etching, make their appearance in the plates or fibres. The orientation of the bodies with respect to each other is different, and for temperatures in the neighbourhood of the meltingpoint a random orientation of the grains is produced; at the same time the grains increase in size, and the last traces of the cold-working vanish.

In continuation of the subject, Dr. Sachs (Frankfurt a. M.) discussed "Problems of the Science of Metals in the case of Aluminium and

No. 3220, VoL. 128] 
Aluminium Alloys", whereby in particular he dealt with the technically important cohesional properties of individual crystals, of rolled alloys, and of cast alloys. Attention was directed to the relations between the plastic properties of metallic crystals and their lattice structure, and emphasis was laid on the differences which exist in the formation of alloys of aluminium as compared with those of copper, silver, and gold. Another aspect of aluminium alloys was discussed, namely, the complicated hardening effects, which may lead to increases by two orders of magnitude in the cohesive properties, as compared with the original modulus of shear of the crystal. Dr. Sachs is inclined to attribute this to changes in the homogeneous phase. Later, the harmful influence of a gas content on cast alloys was discussed theoretically, and finally he considered the process whereby aluminium is protected against corrosion by means of a compact oxide film produced electrolytically.

The last of the general surveys on the subject was presented by Dr. E. Schmid (Berlin-Dahlem). He discussed the "Physics and Metallography of Magnesium ", in which connexion he dealt particularly with those differences which are based on the hexagonal crystal structure of magnesium and its alloys, in contrast to the large group of cubic technical metals.

The president of the Society had not only drawn up the programme of the session at which the five general surveys just mentioned were delivered, but he had also recommended that in the choice of the individual lectures preference should, so far as possible, be given to topics which are related in some way with the main topic of the meeting. Accordingly, in the other sessions at which freely selected individual iectures were delivered, topics taken from the science of metals were in many cases discussed. In view of the large number of these shorter papers-in all about sixty single papers were read-it is only possible here to direct attention briefly to a few of them.

Dr. W. Schmidt (Bitterfeld) dealt with the "Technology and Application of Electron-Metal ", and made detailed statements on the components of this alloy, which is so valuable owing to its lightness, and also on the process of refining and the method of working it. Dr. W. Kaufmann (Frankfurt a. M.) reported on the "Vaporisation of Magnesium in a Vacuum ", and illustrated his remarks by the demonstration of interesting preparations. Dr. Seith (Freiburg) communicated the results of experiments that he had performed in collaboration with Prof. Hevesy on "Diffusion in Metals". The diffusion of a metal in itself (self-diffusion) was treated as a limiting case of the diffusion of one metal in another (foreign-diffusion), and it was shown that in metals self-diffusion is always very slow, large velocities of diffusion only being able to manifest themselves in certain cases of foreign-diffusion. For example, the diffusion of gold in lead at $150^{\circ} \mathrm{C}$. amounts to $4 \times 10^{-3} \mathrm{~cm}^{2}$ day $^{-1}$, whereas lead in lead at the same temperature has a diffusion coefficient of only $8 \times 10^{-10} \mathrm{~cm}^{2}$ day $^{-1}$. Papers by Dr. Pietsch (Berlin) and Dr. Schwab (Munich) dealt with the "Activity of Complex (Mehrstoff) Catalysts" and the question of the active centres in hydration catalysis.

More detailed reference may be made to the lecture by Prof. K. Przibram (Vienna), who presented a very illuminating contribution to the topic of the main lectures, more especially to the lecture of Prof. Tammann; Przibram has succeeded, in a special case, in permanently recording cinematographically the process of recrystallisation.

It is well known that rocksalt assumes a yellow colour under the influence of the rays from radium. The coloration takes place more rapidly and penetrates more deeply when the mineral has been subjected to a uni-directional pressure of more than $100 \mathrm{kgm} . / \mathrm{cm} .^{2}$ before treatment with the rays; rocksalt which has been compressed by a force equal to the weight of a few thousand kilograms becomes almost black in a short time when rayed by means of radium. About two years ago Przibram observed that such specimens, after some time, again show clearer regions, which slowly grow. Since the deeper coloration of the compressed salt was attributable to disturbances of the crystal lattice, it was necessary to conclude that the reoccurrence of clearer patches was due to a progressive healing up of the disturbed lattice, that is, to recrystallisation, a conclusion which could be verified simply by cleaving the specimens, when large reflecting crystal surfaces were revealed as the cleavage planes of the clear patches, whereas the black part showed no cleavage. It could also be proved by means of X-rays that the clear regions again possessed an orderly crystal lattice.

A method thus presented itself of conveniently following up the recrystallisation of the compressed rocksalt, and even of recording it cinematographically by 'stealing a march on time'. The process in the production of the film was the following. A small slab of rocksalt was chosen, which, a few weeks after the above described treatment, revealed a beautifully regular and almost square grain of recrystallisation. Forty exposures of this slab were taken daily-twenty in the morning, one directly after the other, and likewise twenty in the afternoon--and this process was continued for a period of two months. The exhibited film showed first the compressed specimen of rocksalt traversed by cracks, with the above-mentioned clear recrystallised grain in the left upper quadrant. The gradual growth of this grain was clearly to be seen, and it was also evident that the marked horizontal crack separating the two left quadrants provided an insurmountable obstacle to the further growth; the corner of the grain approa $h$ ing it became blunted. Apart from the firstmentioned grain, a number of other grains formed in the course of time, and these likewisecgrew, until finally a large part of the specimen appeared in the recrystallised state.

This first film illustrating recrystallisation is naturally still somewhat defective. There is, how- 
ever, no doubt that when it has been arranged to take the photographs at regular intervals of, say, an hour, day and night, by means of an automatic device, it will be possible to undertake accurate measurements of the velocity of recrystallisation, with the aid of the film. The method is already very useful for the demonstration of the process of recrystallisation.

Mention should be made of a few papers which had no direct connexion with the main theme. Dr. Werner Kuhn (Karlsruhe) presented a communication on "Single and Superposed Absorption Bands and their Behaviour with respect to Optical Activity". With $d$-camphor Dr. Kuhn has found that an absorption band which has usually been considered single is in reality a superposition of a weaker and a stronger band. $\mathrm{He}$ was led to this view by the observation of certain deviations in the behaviour of camphor as compared with that of other optically active compounds. Dr. Fromherz (Munich) spoke on "A Spectroscopic Investigation of the Conditions of Dissociation of the Halides of Metals in Solution". Whereas the absorption curves of light in aqueous solutions of the halides of the alkali and alkaline earth metals are essentially independent of the concentration as regards their shape and position, up to the highest concentrations, new absorption bands of association products make their appearance alongside the absorption bands of the hydrated ions in aqueous solutions of lead, thallous, cadmium, and mercury halides, and they arise to an increasing degree as the concentration of the solutions is increased. From the spectral position of the bands and the dependence of their height on the concentration of the contributing ions, it has been possible to determine the nature of the association products, the dependence of the degree of association on the concentration, and hence the equilibrium of association in the solutions. Dr. Beutler (Berlin) gave an account of a piece of work which was concerned with dissociation as a result of collisions of the second kind, and in which, in particular, the splitting up of the hydrogen molecule into a normal and an excited hydrogen atom by means of excited neon atoms was followed up spectroscopically. Prof. W. A. Noyes (Urbana) pointed out that the formula proposed by Mecke on the basis of spectroscopic data for nitrogen dioxide $\mathrm{O}=\mathrm{N}-\mathrm{O}-$ is in good agreement with the views on 'shared' and ' unshared' electrons which have been derived from the chemical behaviour of nitrogen dioxide.

Dr. Frankenburger (Ludwigshafen a. Rh.) has continued with his collaborators the attempts to combine hydrogen or nitrogen with highly disperse metals. Their method is to separate iron or nickel from the vapour phase; the resulting metals, which are always at first in an extremely finely divided form, join up to form larger particles if this process of collective crystallisation is not suitably prevented. Frankenburger realises this by arranging that sodium chloride is deposited from the vapour phase simultaneously with the metal. When the molecular ratio $\mathrm{NaCl}$ : $\mathrm{Fe}$ was increased to 2000 or 3000 , it was found possible to combine up to six molecules of hydrogen with each atom of the separated iron. We are here obviously dealing with weak affinities between metal atoms and gas molecules, forces akin to those of van der Waals in the gas theory. In the discussion, Prof. Biltz (Hannover) suggested that the phenomenon is perhaps in its nature related to the attachment of five or six molecules of water of hydration to the chemically non-reactive atoms of argon, krypton, and xenon.

Prof. Paneth (Königsberg) reported on the continuation of the investigations on free methyl and ethyl, which were intended to bring clarity with regard to the mechanism of the disappearance of the free radicals. If helium instead of hydrogen is used for the transport of the radicals, their activity falls off with the same velocity as in hydrogen; from this and from related observations it is concluded that a reaction takes place with the vessel walls. Whereas in a glass tube the radicals can be transported more than half a metre by a rapid gas flow, a metal mirror $1 \mathrm{~cm}$. in length introduced into the tube suffices to bind the radicals quantitatively. On the basis of formulæ derived from the kinetic theory of gases by Prof. K. F. Herzfeld (Baltimore), we can calculate from these results that every free radical is bound by a singleimpact on a metal capable of reaction, whereas from amongst one thousand radicals incident on a glass wall only about one is not reflected. From the discussion on this paper we may direct attention especially to the remarks of Prof. Bodenstein (Berlin), who mentioned similar observations in the combination at glass walls of $2 \mathrm{Cl}$ to form $\mathrm{Cl}_{2}$.

It was decided to hold the next meeting of the German Bunsen Society at Münster, in the year 1932.

\section{Obituary.}

Srr Hugh Ball, Bart., C.B.

TWHE north-east coast of England will have bitter 1 reason to remember for long the first half of the current year, because this highly industrial corner of England has lost by death a greater number of its leading industrialists than can be remembered in any similar period. The names of such men as Sir Arthur Dorman, Sir Charles Parsons, Andrew Laing, Sir Archibald Ross, and now Sir Hugh Bell, all dead since the beginning of the year, show how severely it has suffered; such losses would be serious enough at any time, but at a time of crisis like the present, when men of experience, judgment, and knowledge are so badly needed, the loss is even exceptionally deplorable: for, as the above names show well enough, these were men who were known and honoured not only in their own district but also in all industrial circles throughout the world, and no one was better known or more highly honoured than the man whose loss we mourn to-day. 